\title{
Research on Parametric Form Design Based on Natural Patterns
}

\author{
$\mathrm{X} \mathrm{Li}^{1}, \mathrm{JN} \mathrm{Su}^{2, \mathrm{a}}$ \\ ${ }^{1}$ School of Mechanical \& Electronical Engineering, Lanzhou University of Technology,730050 Lanzhou Gansu, China \\ ${ }^{2}$ School of Design Art, Lanzhou University of Technology, 730050 Lanzhou Gansu, China
}

\begin{abstract}
Parametric form design method based on natural patterns is proposed for the design schema of the traditional form bionics. Firstly, the concepts of natural patterns are analyzed, concluded and summarized. Secondly, the parametric design thinking is elaborated from three aspects: thinking mode, design flow, tools and scripts. It is also proposes a parametric logo design method. This paper takes logarithmic spiral pattern as an example to describe the process from the law of natural pattern to logo design, which includes nature pattern analysis, control rules of from and color, algorithm research and generating design.
\end{abstract}

\section{Introduction}

The aesthetic laws of statistical aesthetics, most of which are based on the structure and growth of plants and animals in the natural world ${ }^{[1]}$. Learning in nature exists at every stage of design development. Different stages of development have different understandings. From the initial appearance simulating to rational creation born out of the inside, humans gradually increase the integration of design and nature. Design absorbs nourishment from nature and helps to create beauty actively and regularly, including new forms and new structures. The design pursues the realm of natural harmony between man and nature.

Parametric technology has been widely used in architectural design, industrial product design, landscape design, fashion design, jewelry design and other design fields ${ }^{[2]}$. The goal of parameterization is to construct an automatic design system that can be edited at any time. Parametric technology provides infinite creativity for $\operatorname{design}^{[3]}$. It can obtain several design schemes at one time, thus improving the design efficiency.

For the design schema of the traditional bionic form, this paper focused on the mathematical logic behind natural patterns, and studied the parametric form design method based on natural patterns.

In the following Section II, we provide a brief introduction to the concept of natural pattern, including analysis and induction. Section III discusses the parametric design, including process, thinking model, tools and scripts. Section IVstudies the method of parametric logo design. Case study in Section V. The last part is the conclusion.

\section{Natural Pattern}

*Corresponding author: ${ }^{\text {a }}$ sujn@lut.cn
The term pattern is not unfamiliar and covers a wide range of fields, such as biology, economics, computer science and technology, physics.

We are in nature. Human exploration of natural patterns began as early as ancient Greek philosophers. Plato, Pythagoras, and Empedocles had tried to reveal the order of the natural world, and established the philosophical foundation for the study of natural patterns. In 1202, Leonardo Fibonacci published his famous book Liber Abaci, and raised the Fibonacci numbers on the issue of rabbit breeding ${ }^{[4]}$. In the 19th century, the Belgian physicist Joseph Plateau studied the soap film, prompting him to propose the concept of minimal surface [5]. The Scottish biologist and molecular mathematician D'Arcy Thompson took the lead in researching the growth patterns of plants and animals. In 1917, he published a book On Growth and Form. In his book, Thompson proposed to associate phyllotaxis with the Fibonacci sequence ${ }^{[6]}$. He showed that complex spiral growth can be explained by simple equations. In the 20th century, the British mathematician and the "father of artificial intelligence" Alan Turing pushed the study of natural patterns to a climax. In 1952, he wrote The Chemical Basis of Morphogenesis ${ }^{[7]}$, and the seemingly complex and irregular pattern of spots and stripes could be described by the Turing equation. Then, in 1968, Hungarian biologist Aristid Lindenmayer developed the L- system to simulate the fractal of plant growth pattern ${ }^{[8]}$. The scientific research results of these natural patterns are the premise of 'design research based on natural patterns'.

Patterns in nature are visible regularities of form found in the natural world ${ }^{[6]}$. Symmetry patterns are ubiquitous in nature. Humans and animals are mainly mirror-symmetrical and plants usually have radial or rotational symmetry. Non-living bodies also have symmetrical patterns, such as snowflakes, ice crystals, crystals and so on. Spirals is undoubtedly the most 
beautiful universal pattern, the initial pattern of life system. From the inanimate spiral galaxy to the genetic material of life the double helix of DNA molecule, petals, fingerprints, eddy currents, cochlea, the tendrils of plants and so on. Insects and animals show the extremes of the spots and stripes pattern. For example, leopards, cows and ladybugs are full of spots, zebras and angelfish have distinctive stripes, butterflies blend spots and stripes. Meanders are common in nature and life, such as the whereabouts of animals, brain gyrus, artificial mazes, and pedestrian trails. Branches and fractals are widespread in nature and have infinite self-similarity. Such as lightning, mountains, coastline, the body's nervous system and blood vessels branch, etc. Alongside fractals, chaos theory ranks as an essentially universal influence on patterns in nature. Mathematics tries to discover and explain various abstract patterns and rules. The visual model of nature can find explanations in logarithmic spirals, fractals, topology, chaos theory, and other mathematics, which lays a mathematical foundation for parametric design.

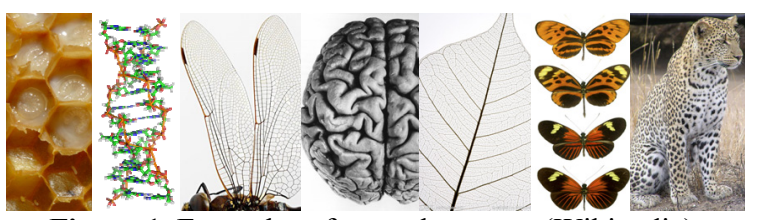

Figure 1. Examples of natural patterns (Wikipedia).

Patterns organizes and defines the relationship of nature, and can be applied to practical design to enhance and support visual communication ${ }^{[6]}$. This article attempted to develop the intersection between natural patterns and design sciences, and tried to combine natural patterns with the form design through computer programming techniques to explore a new form-aided design approach.

\section{Parametric design}

Parametric design originated from mechanical design ${ }^{[9]}$. The fundamental difference in parametric design is the application of computer programming techniques, compares to a typical sketch-manual modeling design schema. Its purpose is to construct an automated generative design system that can edit and modify parameters at any time. Therefore, the parametric design has changed in three aspects: Design Processes (DP), Design Thinking (DT) and Design Tools (DT). This also leads to the design cognitive model of designers to be changed.

\subsection{Parametric design thinking(PDT)}

Hugh Whitehead thinks that parameterization is more about a way of thinking ${ }^{[10]}$. Rivka Oxman ${ }^{[11]}$ claims that this is mainly due to the intersection of three areas of knowledge: professional design knowledge, computer programming knowledge, and mathematical knowledge. Parametric design does not deal directly with the form, but rather studies the mathematical logic behind the form.
Computer programs are used to calculate the various elements that affect the styling (parameter variables).

Robert Woodbury ${ }^{[10]}$, in his book Elements of Parametric Design (2010), proposed that Parametric Design Thinking(PDT) has three main characteristics abstract thinking, mathematical thinking, algorithmic thinking. Parametric design thinking processes shown in Figure 2 can also prove this view. Abstract thinking is the basis of parametric design, abstracting design ideas or concepts into symbols, data, variables, functions, etc. Mathematical thinking mainly involves how to translate mathematical theorems and data structures into useful algorithms ${ }^{[12]}$. The essence of algorithmic thinking is the step-by-step problem solving. In parametric design, algorithmic thinking means writing functions in scripting languages. Functions are part of the digital form, the editing function is to edit digital form.

In traditional paper-based design, designers mostly reduce the distance from the ideal design scheme by drawing sketches and making models (digital or physical models). At the beginning, however, parametric design schema need to transform concepts into data. Compared with traditional paper-based design, parametric schema emphasizes systematic design process and the geometry logic of form.

The organic management of data is the key for designers to form designs by programming technology. That is: data flow driven form, data structure organize form. All of this is accomplished by data transfer between the various functions written in a scripting language. In computers, form is represented by data. Designers achieve design goals through 'design' code while the code records the thought process of the designer. The design process of the human brain is no longer a black-box operation.

Therefore, the parametric thinking schema can be understood as a process of integrating abstract thinking, mathematical thinking and algorithm thinking in order to realize the design goal of organic automation under the guidance of design knowledge.

\subsection{Parametric design process}

Whether there is a specific thinking process in parametric design, there is no definite conclusion yet ${ }^{[12]}$. However, no matter what type of design has its own general design process. Figure 2 is a typical parametric design thinking process. First of all, consider concepts and ideas based on customer needs and design tasks, and present them in sketches, text, or data. Secondly, designers constantly abstract and digitizes ideas and concepts, explore the geometric logic rules, and include evaluation rules ${ }^{[13]}$. Gradually, designer establish an associative set of algorithmic rules and data structure. In the third step, the designer must standardize all parameters, including the definition, coding, and naming of the parameters, and further clarify the relationship between the design input and the design response (design output $)^{[3]}$. Further, designers need to consider the internal data stream transfer parameters to form a control parameter of the organic system. Step Four: designer 
writes the script code in the script environment according to the algorithms, rules and data structure, runs and debugs the program, and checks the design response. Finally, the designer evaluates the output and communicates with the customer to provide timely feedback. However, the design is not a linear process, especially the process of parameterization. Modifying rules, algorithms, and code is a regular job.

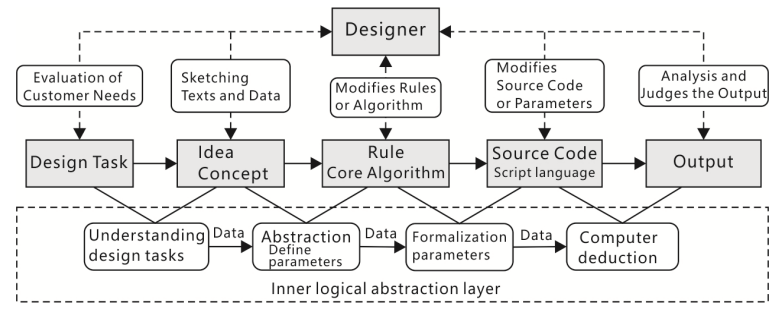

Figure 2. The process of parametric design.

\subsection{Parametric design tool and scripts}

The core work of parametric design is algorithm research and programming. The design of parametric digital form by programming technology requires graphic design platform and script environment. For example, CorelDraw is a graphic design platform, VBA can be used as a scripting language. However, this is not a secondary development in the general sense, which is a branch or direction of design method and design technology development. In script environment, form is driven by algorithm and code. Program statements and the definition of functions and classes can express the form logic construction process ${ }^{[14]}$.

Grasshopper is the most popular parametric design tool, and is a visual scripting language. It has been built into Rhino6.0. Grasshopper itself supports multiple scripting languages such as Python, C\#, and VB. It is often used in architecture design, industrial design, jewelry and so on. Illustrator supports multiple scripting environments (VB, JavaScript, AppleScript). Processing is an extension of the Java language, which can be used for visual communication design, interactive media art design, and information visualization ${ }^{[15]}$, such as the logo designed by the Danish design studio NR2154 for United Nations Climate Change Conference in Copenhagen. Mathematica and Matlab can be used for the calculation of parametric form design ${ }^{[16]}$. The features of parametric tools and scripts are listed in Table 1.

Table 1. Tools and scripts for parametric design.

\begin{tabular}{|c|c|c|}
\hline Tools and scripts & $\begin{array}{l}\text { Language type/ } \\
\text { Futures }\end{array}$ & Scope of application \\
\hline $\begin{array}{c}\text { Rhino / } \\
\text { Grasshopper } \\
\text { /Python }^{[14]}\end{array}$ & $\begin{array}{l}\text { Visual scripting code / Code } \\
\text { and model in parallel }\end{array}$ & $\begin{array}{l}\text { Architecture/ } \\
\text { Product etc. }\end{array}$ \\
\hline $\begin{array}{l}\text { CorelDRAW / } \\
\text { VBA }^{[17]}\end{array}$ & Macro scripting language & graphic design \\
\hline $\begin{array}{l}\text { Illustrator/ } \\
\text { JavaScrip }^{[18]}\end{array}$ & scripting language & graphic design \\
\hline $\begin{array}{c}\text { Processing } \\
(\text { JavaScrip })^{[15]}\end{array}$ & Graphic design language & $\begin{array}{c}\text { Interactive/Visual } \\
\text { Art/Information } \\
\text { Visualization }\end{array}$ \\
\hline $\begin{array}{l}\text { Mathematica } \\
(\text { wolfram })^{[19]}\end{array}$ & symbolic language & $\begin{array}{c}2 \mathrm{D} / 3 \mathrm{D} \\
\text { design calculation }\end{array}$ \\
\hline
\end{tabular}

The parametric algorithmic schema has been completely different from the traditional paper-based design schema. However, this does not mean that the designer must become a programmer, or that the programmer can replace the work of designer. In the process of parametric design, therefore, designers need to constantly balance programming techniques and the understanding of design knowledge ${ }^{[11]}$.

\section{The method of parametric logo design}

As a typical form, the logo is the core of enterprise brand image. A good logo is both attractive and meaningful. It is an important bridge between the public and the company. The core steps of the parametric logo design based on the natural mode are as follows.

Firstly, designers must have a deep understanding of the culture, market environment and core values of the enterprise, and extract core demand and key visual elements ${ }^{[20]}$.

Secondly, we need to find natural patterns that match the design requirements. The natural patterns that match the enterprise image can be crossed and merged in several natural patterns. For instance, symmetry and rotation can be fused with spiral pattern. After the match is successful, the visual constituent laws and associated logic of the selected natural patterns can be studied, especially the geometric logic relations. At this point, designer can initially define the parameter set of the logo form: $\left\{s_{1}, s_{2}, s_{3}, \ldots, s_{n}\right\}$.

Then, according to the geometrical logic relation and the form association logic, designers can construct the transformation rule of the form, and transform all the rules into the transform algorithm of the logo form. On the other hand, the overall image of the logo is also affected by other factors, such as proportion, location, text, etc. This requires the construction of some additional rules for systematic coordination. In addition, designers should define color control parameter set: $\left\{c_{l}\right.$, $\left.c_{2}, c_{3}, \ldots, c_{n}\right\}$ and color transformation rules based on the color design scheme of the logo and color space. In order to facilitate the color transformation and algorithm implementation, it is necessary to map the value range of the color space to the interval of $[0,1]$. Sometimes, in order to show better results, it is necessary to associate the color parameters of the logo with the form parameters of the logo.

Finally, according to design requirements, we need to define the font control parameter set: $\left\{f_{1}, f_{2}, f_{3}, \ldots, f_{n}\right\}$ and font control rules.

The process of studying natural patterns is also the process of studying algorithms. Parametric form design is a process of parametric experiment. It constantly adjusts rules and modifies parameters to satisfy the visual demand of the customer based on design responses.

\section{Case study}

The design task from Gansu ZBloom Culture Media Co. Ltd. and its core philosophy: the culture spread to the 
hearts of the people, the spirit of sustainable artisans. The spiral pattern is radially scattered from the center to the outside, implying the infinite transmission of the energy of cultural media, which coincided with the core philosophy of ZBloom Cultural Media. This case, therefore, applied a logarithmic spiral pattern to the parametric form design.

\subsection{Logarithmic spiral pattern}

Logarithmic spiral, also known as isometric spiral or growth spiral, is a self-similar spiral curve and it is a common pattern in nature. Logarithmic spiral was first described by Descartes and later extensively investigated by Jacob Bernoulli, who called it 'the marvelous spiral' $^{[5]}$. The Fibonacci spiral also known as the 'golden spiral' in geometry. It is a special case of logarithmic spiral and is also the most perfect classic gold proportion in nature. The logo design of this case used a logarithmic spiral pattern which comes from Nautilus and seed head of sunflower (Figure 3).

The head of sunflower is spiral. Its seeds are Fibonacci spiral arrangement, and there are two groups of spiral in the opposite direction. This arrangement is described by any spiral, wherein the spiral of Archimedes easiest. The definition of Archimedes spiral line: $\mathrm{r}(\theta)=\mathrm{a} \theta$ (polar equation). Generally, there are 34 to 55 spiral lines from inside to outside. 34 and 55 are two consecutive numbers in the Fibonacci sequence.
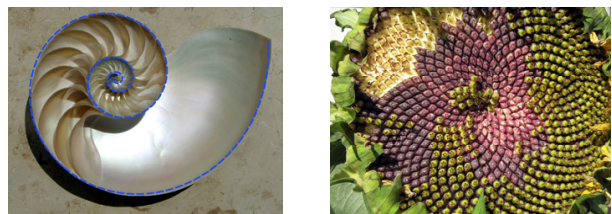

Figure 3. The classical logarithmic spiral in nature: Seed head of sunflower and Nautilus (Wikipedia) is

The polar coordinate definition of logarithmic spiral

$$
\mathrm{r}(\theta)=\mathrm{ae}^{\mathrm{b}} \theta
$$

where $\mathrm{a}, \mathrm{b} \in \mathrm{R}$, and $a \neq 0$. In order to facilitate the implementation of the algorithm, the formula (1) must be converted into a parametric equation as shown in formula (2).

$$
\left\{\begin{array}{l}
x(t)=r(t) \cos (t)=\mathrm{a} e^{\mathrm{b} t} \cos (t) \\
y(t)=r(t) \sin (t)=\mathrm{a} e^{\mathrm{b} t} \sin (t)
\end{array}\right.
$$

\subsection{The control rules of form and color}

The parametric design of the logo includes three aspects: parametric form, parametric color, and parametric text. In this case, we used disks instead of sunflower seeds for parametric logo design.

The sine function was selected as the radius transformation function of disks. The radius changes with the number of disks, but the radius of the disk cannot be negative. So we defined the disk radius transformation function as shown in formula (3).

$$
\operatorname{Disks} \operatorname{Radius}(n)=\left\{\begin{array}{r}
\mathrm{a} \sin (\mathrm{b} n)+\mathrm{c} \text { if } n \geq 0 \\
0 \text { if } n<0
\end{array}\right.
$$

where $\mathrm{a}, \mathrm{b}$ and $\mathrm{c}$ are positive real numbers, and $\mathrm{a} \leq \mathrm{c}$. The independent variable $n$ is the number of disks, $n \in[0,+\infty)$. The function image is shown in Figure 4.

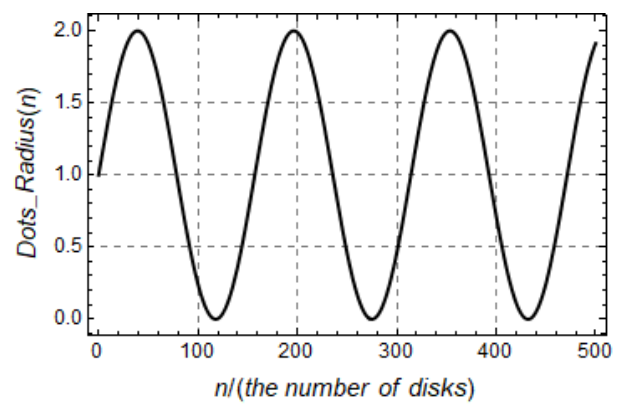

Figure 4. Function image of the disks radius transform.

RGB, HLV, CMKY and other color spaces can be parameterized by logo colour. The value of the color space was converted to $[0,1]$ according to the script environment and programming needs. Therefore, the color transformation function can be defined by the hyperbolic tangent function, which is the quotient of the hyperbolic sine and the hyperbolic cosine. Its definition process is as follows:

$$
\begin{gathered}
\sinh (x)=\left(\mathrm{e}^{x}-\mathrm{e}^{-x}\right) / 2 \\
\cosh (x)=\left(\mathrm{e}^{x}+\mathrm{e}^{-x}\right) / 2 \\
\tanh (x)=\sinh (x) / \cosh (x)=\left(\mathrm{e}^{x}-\mathrm{e}^{-x}\right) /\left(\mathrm{e}^{x}+\mathrm{e}^{-x}\right)
\end{gathered}
$$

From the formula (6), we have $x \in(-\infty,+\infty)$ and $\tanh (x) \in(-1,+1)$. But the color values cannot be negative. Therefore, we defined the following function:

$$
\text { Color_Transform }(n)=\left\{\begin{aligned}
\tanh (\mathrm{k} n) & \text { if } n \geq 0 \\
0 & \text { if } n<0
\end{aligned}\right.
$$

where $\mathrm{k} \in R^{+}, n \in[0,+\infty)$, and the independent variable $n$ is the number of disks. The value of constant $\mathrm{k}$ determines the quality of the color transformation. The process of the color change is shown in figure 5.

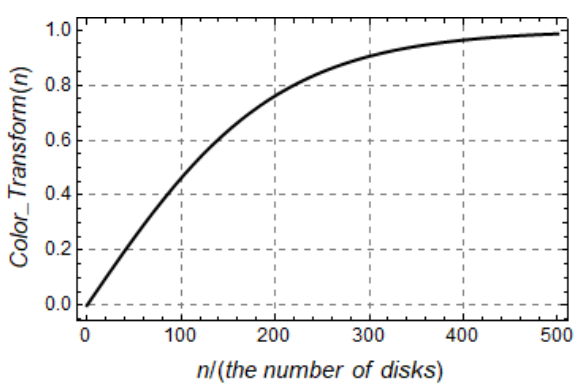

Figure 5. Function image of color transformation.

The combination of the blending colour algorithm and the color transformation function can present 
multicolour nonlinear gradient of the color design. In this case, the mixture of the three colors is defined as the color scheme of the logo.

\subsection{Core algorithm}

Firstly, the logarithmic spiral arrangement of disks is implemented according to formula (2), where the parameters are the radius and the angle. $[0, \mathrm{a}+\mathrm{c}]$ is the domain of the radius changes of disks, where a and $\mathrm{c}$ are constants in equation (3). $\left[0^{\circ}, 360^{\circ}\right]$ is the domain of angle changes. Secondly, according to the formula (3) to achieve the control algorithm of the change of disks radius, the range of the number of disks is $[0, \mathrm{~N}]$, where $\mathrm{N}$ is a constant. The constant $\mathrm{N}$ is determined according to the design response and can be modified at any time. Thirdly, three color nonlinear gradient design is achieved by the blending colour algorithm and the formula (7). The color change of the logo with the number of disks $\mathrm{n}$ change. Finally, we can achieve the function of calling and selecting fonts by calling the standard font of the Window system and the Font library of the script environment.

\subsection{The generator of parametric logo design}

This article developed a generator of parametric logo design (Figure 6) with Wolfram language in mathematica for Gansu ZBloom Cultural Media. The left side of the logo generator interface is the parameter control bar of the logo, and the right side is the logo browser. The form parameters of the logo includes four parameters: the angle, the radius of disks, the number of disks, and the size of the logo image.

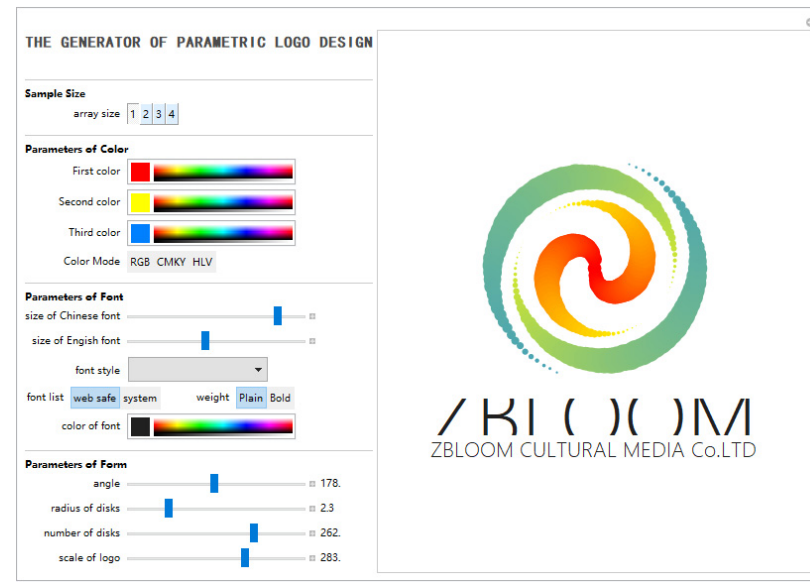

Figure 6. The generator of the parametric logo design.

Design results in Figure 7 selected out by the designers, which were selected in the first round. They were designed by the logo generator under different parameter values. The parameter values are shown in Table 2. Figure 7(c) was selected as the logo for the ZBloom Cultural Media judged by a evaluation group.

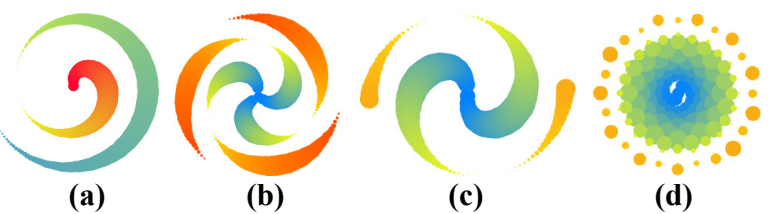

(a)

(b)

(c)

(d)

Figure 7. The forms of logo in different parameters.

Table 2. Parameters of the logo form

\begin{tabular}{|c|c|c|c|c|c|}
\hline $\begin{array}{c}\text { Para } \\
\text { meter } \\
\text { s }\end{array}$ & angle & $\begin{array}{c}\text { Min radius } \\
\text { Max radius }\end{array}$ & $\begin{array}{c}\text { Number } \\
\text { of disks }\end{array}$ & $\begin{array}{c}\text { Image } \\
\text { size }\end{array}$ & $\begin{array}{c}\text { Color value } \\
\text { (RGB) }\end{array}$ \\
\hline (a) & 2.3 & $\begin{array}{c}0.1 \\
1.75\end{array}$ & 271 & $390^{2}$ & $\begin{array}{c}(255,0,50) \\
(230,255,30) \\
(20,128,155)\end{array}$ \\
\hline (b) & 119 & 0.31 & 261 & $272^{2}$ & $\begin{array}{c}(15,128,253) \\
(250,255,15) \\
(255,0,50)\end{array}$ \\
\hline (c) & 178.33 & 0.25 & 164 & $374^{2}$ & $\begin{array}{c}(162,0,0) \\
(255,255,23) \\
(10,150,210)\end{array}$ \\
\hline (d) & 195 & 0.6 & 165 & $348^{2}$ & $\begin{array}{c}(0,128,255) \\
(255,255,10) \\
(240,20,45)\end{array}$ \\
\hline
\end{tabular}

\section{Conclusions}

Natural patterns has provided an unlimited source of design and can be effectively applied to form design. Parametric design can effectively enrich the content of form design. Finding the mathematical logic behind natural patterns has been a key step. The combination of natural patterns and parametric design technology will open up new design schema and methods for form design. This has been a fusion of aesthetics and technology. Parametric languages and scripting techniques will have an unprecedented impact on design thinking. Designers has been required to constantly update their knowledge structure. Parametric design has required designers to respect the mathematical and logical laws of the scripting environment. It has been difficult for designers to anticipate design results in a scripting environment. The combination of natural patterns and parametric thinking has prompted the form design becoming an generative system of organic closedloop from design requirements to design results.

The next work of this article will study the parametric design method of complex product form based on natural patterns.

\section{Acknowledgments}

The project is sponsored by National Natural Science Foundation of China (51465037) and Hongliu Outstanding Talent Development Program of Lanzhou University of Technology (201406). In addition, Zbloom Culture Media also provided support for this project.

\section{References}

1. WJ Yue. The natural theorem of art and design. Shanghai Art \& Crafts, 1, 82-83 (2011) 
2. J Chen. Research and practice of product design based on parameterization. Art Journal, 3, 111-115 (2015)

3. WG Xu. Parametric design and algorithm generating. World Architecture, 6, 110-111 (2011)

4. DL Ma. A study on Fibonacci's Liber Abaci (PhD thesis). (ShangHai Jiao Tong University, Shanghai, 2009)

5. S Hildebrandt, A.J Tromba, $\mathrm{S}$ Sheng. The Parsimonius Universe. (ShangHai Education Press, Shanghai, 2004)

6. M Macnab, WB Fan. Design by nature: using universal forms and principles in design (China Machine Press, Beijing, 2013)

7. AM Turing. The chemical basis of morphogenesis. 1953. Bull Math Biol, 52,153-197(1990)

8. A Lindenmayer. Mathematical models for cellular interactions in development. Journal of Theoretical Biology, 18, 3, 280-315 (1968)

9. CRB Hernandez. Thinking parametric design: introducing parametric Gaudi. Design Studies, 27, 3, 309-324 (2006)

10. R Woodbury. Elements of Parametric Design (Routledge, New York, 2010)

11. R Oxman, $\mathrm{N}$ Gu. Theories and Models of Parametric Design Thinking. eCAADe 33, 2, 477482 (2015)

12. $\mathrm{R}$ Oxman. Thinking difference: Theories and models of parametric design thinking. Design Studies, 52, 4-39 (2017)

13. YC Liu. Parametric Design: Method, Thinking Strategy and Framework. Architecture Technique, Z1, 34-37 (2011)

14. RQ Bao. Parametric Logic Design Process (Phoenix Science Press, Nanjing , 2015)

15. L Tan. Application Research of Code Art Based on Processing. Art \& Design, 2, 104-105 (2012)

16. JN Su, JH Wu, Y Liu, QW Zhang. Research on 3D Product Shape Merging Technology Based on Spherical Harmonic Map. Journal of Graphics, 35, 3, 417-422 (2014)

17. XJ Liu, YY Wu. The Research on Rapid Design of Fan Products by Computer-Aided Intelligence. Packaging Engineering, 10, 42-45+56 (2013)

18. M O'Neill, A Brabazon. Evolving a logo design using Lindenmayer systems, Postscript \& Grammatical Evolution. Evolutionary Computation. IEEE, 3788-3794 (2008)

19. S Wolfram. An Elementary Introduction to the Wolfram Language (Wolfram Media Inc, Champaign, 2016)

20. DQ Jin. The Brand Design 100+1 (Peking University Press, Beijing, 2017) 